Meta

Journal des traducteurs

Translators' Journal

\title{
Les drogmans de Malaisie
}

\section{Laurent Metzger}

Volume 38, numéro 3, septembre 1993

URI : https://id.erudit.org/iderudit/003643ar

DOI : https://doi.org/10.7202/003643ar

Aller au sommaire du numéro

Éditeur(s)

Les Presses de l'Université de Montréal

ISSN

0026-0452 (imprimé)

1492-1421 (numérique)

Découvrir la revue

Citer cette note

Metzger, L. (1993). Les drogmans de Malaisie. Meta, 38(3), 575-576.

https://doi.org/10.7202/003643ar

Ce document est protégé par la loi sur le droit d'auteur. L'utilisation des services d'Érudit (y compris la reproduction) est assujettie à sa politique d'utilisation que vous pouvez consulter en ligne.

https://apropos.erudit.org/fr/usagers/politique-dutilisation/
Cet article est diffusé et préservé par Érudit.

Érudit est un consortium interuniversitaire sans but lucratif composé de l’Université de Montréal, l'Université Laval et l'Université du Québec à Montréal. Il a pour mission la promotion et la valorisation de la recherche. https://www.erudit.org/fr/ 


\section{LES DROGMANS DE MALAISIE}

La traduction du français au malais et vice-versa n'a pas atteint sa vitesse de croisière. Certes elle ne date pas d'hier, puisqu'un grand nombre d'orientalistes français nous ont précédés dans la carrière et ont effectué; au siècle dernier, plusieurs traductions pour faire connaître, au public français, la richesse de la littérature classique malaise. Comme la liste de ces traducteurs est assez longue, il suffira d'en évoquer quelques représentants, tels Édoudard Dulaurier, le père des études malaises en France, Aristide Marre, Marcel Devic, Alfred Tugault, etc.

Un nouvel élan est apparu dans les années 1980 dans les deux sens, français-malais et malais-français. C'est en effet à partir de 1982 que plusieurs romans malais ont pu être lus par nos compatriotes par le biais des traductions. Ainsi S. Othman Kelantan, Shahnon Ahmad, Anwar Ridhwan et aussi un certain nombre d'auteurs de nouvelles ont pu être lus, et appréciés, en français. Parallèlement donc, plusieurs cuuvres françaises ont été mises à la portée des lecteurs de Malaisie. Des auteurs variés ont pu de cette façon avoir des lecteurs malaisiens. Maupassant, Roland Barthes, Jean-Jacques Rousseau, Georges Mounin ont reçu un auditoire supplémentaire par le truchement de la traduction en malais.

Néanmoins tout n'est pas parfait - et loin de là - dans cette mise à la portée du pays cible d'une littérature étrangère. Les traductions du siècle dernier étaient en particulier bien souvent partielles, sinon partiales. Il faut leur reconnaître en tous cas une qualité, celle d'avoir été accomplies et, du reste, souvent d'avoir précédé certaines traductions (certes plus complètes, mais plus tardives en langue anglaise ou hollandaise). Celles de ce siècle semblent assez disparates, non que la qualité du travail soit mis en cause, mais - dans les milieux bien informés - on constate et on semble déplorer plutôt une trop grande variété dans les choix proposés aux lecteurs cibles. Alors on a songé à la constitution de programmes de traductions et plusieurs listes ont ainsi été proposées à la grande maison d'édition nationale de Malaisie, le Dewan Bahasa dan Pustaka. Tout d'abord le Bureau d'action linguistique, à l'époque où celui-ci était dirigé par Pierre Mattot a présenté une première liste à cet établissement, puis René Daillie, du temps où ce dernier était en mission au Dewan Bahasa dan
Pustaka, en a fourni une autre, Pierre Labrousse de l'INALCO de Paris, auquel on s'était adressé, en a compilé une autre, enfin votre serviteur, qui lui aussi s'est intéressé à cette question, a fait de même et a donc soumis au DBP, une autre liste. Le résultat de tout ceci a été que ces listes étaient différentes et en fait fort différentes. On doit tout de suite comprendre que le contraire eût été surprenant. Il est donc tout à fait naturel que ces listes soient différentes les unes des autres. Une première question se pose alors. Doiton proposer une liste rigide et $\mathrm{s}$ 'en tenir à cette liste, ou tenter de faire autre chose? Nous voudrions ici rappeler un propos de l'éditeur Hubert Nyssen, qui a eu - entre autres - la particularité de s'installer en province, d'y rester et de prouver ainsi qu'on pouvait réaliser quelque chose en dehors de la capitale française : à la question de savoir ce qu'il publiait, il a répondu (bien sûr c'était au début de l'aventure d'Actes Sud, sa maison d'édition d'Arles) qu'il cédait souvent à des coups de cœur. Dans le domaine de la traduction, doit-on proposer une liste étroite et fermée et ne s'en tenir qu'à cette liste? Nous ne le pensons pas, et les coups de cour doivent également intervenir dans cette activité. Ainsi au lieu de ne proposer que quelques choix aux traducteurs (ou aux traducteurs potentiels) il est préférable de leur laisser carte blanche. À eux donc de déterminer quel texte ils devraient proposer à leurs lecteurs. C'est le traducteur qui doit être le chercheur, le prospecteur, le découvreur du texte étranger susceptible d'intéresser les lecteurs du pays cible. Tout le monde sait très bien qu'on traduit mieux ce qu'on apprécie et ce qu'on aime, qu'un texte imposé et qui ne nous plaît guère.

Au-delà du choix du texte, qui doit donc être laissé au traducteur, d'autres embûches apparaissent sur le chemin étroit de la traduction. Ainsi le traducteur a choisi une œuvre, a passé de longs moments à lire et à traduire et alors il se met en quête (s'il ne l'a pas encore fait) d'un éditeur. La chose n'est pas aisée et notre traducteur, qui s'est beaucoup dépensé et fait ceuvre de pionnier, ne trouve pas facilement un éditeur qui accepte de publier sa traduction. Le traducteur est enfermé dans un cercle vicieux. Il tente de faire connaitre un exemple d'une littérature peu connue en France - en fait, que connaissons-nous en France des littératures d'Asie? Bien sûr, nous disposons d'une série de traduction d'œuvres chinoises; nous avons aussi, dans une moindre mesure, des 
textes japonais, eux-mêmes le fruit d'une seconde traduction, de l'allemand ou de l'anglais avant d'être traduits en français, mais lisons-nous des cuvres indiennes, birmanes, thaillandaise, etc.? - et le traducteur se heurte à la prudence ou à la crainte de l'inconnu de l'éditeur, qui n'ose pas proposer une œuvre d'un domaine inconnu sur le marché. Alors la traduction fera longtemps antichambre et votre serviteur en sait quelque chose, puisqu'un texte que j'ai traduit du malais, il y a près de vingt ans n'est toujours pas publié. L'éditeur, si parfois il manifeste un certain intérêt pour ce texte nouveau, propose parfois des coupes, et des coupes sombres. Doit-on les accepter et se dire qu'il est préférable de faire connaître ce texte - même abrégé - que de refuser la moindre modification? Les cuvres littéraires sont parfois fort longues, mais elles sont acceptées en entier par les éditeurs (nous songeons en particulier aux romans russes ou à d'autres œuvres d'Amérique latine). En fait le problème des coupes se pose dans les deux sens, même si c'est pour des raisons différentes. Les éditeurs occidentaux veulent des textes rapides, qui évitent les répétitions, relativement fréquentes en malais; alors les textes sont réduits après avoir été traduits. Est-ce la bonne voie à suivre? Doit-on se plier à nos goûts habituels et rejeter ou modifier tout ce qui nous est un tant soit peu inhabituel ? Grâce à la traduction, pouvons-nous introduire un nouveau produit sur le marché, ou devons-nous l'adapter pour ménager les susceptibilités, les craintes et souvent l'ignorance de ceux qui décident des publications sur le marché local? C'était l'idée maîtresse que j'avais lancée au Centre East-West de Honolulu en janvier dernier, lors d'une conférence internationale sur la traduction, où j'avais prononcé une conférence intitulée : «La traduction : Adaptation ou importation culturelle ?» J'avais choisi ce titre et ce sujet à la suite de déboires avec un éditeur malaisien qui avait tronqué ma traduction d'une cuvre destinée à la jeunesse. Quatre petits chapitres de cet ouvrage avaient été supprimés par l'éditeur, qui arguait qu'il ne pouvait publier un chapitre qui évoquait l'amitié d'un enfant et d'un chien en pays musulman et un autre où il était question d'enfants qui faisaient l'école buissonnière. Dans les deux exemples ci-dessus - nous pourrions multiplier les exemples - l'éditeur redoutait les foudres des autorités, s'il publiait l'ouvrage tel quel. N'est-ce pas fort regrettable? Que faire dans une telle situation? Naturellement j'avais tenté — en vain de plaider ma cause auprès de cet éditeur, en lui expliquant que la jeunesse avait besoin d'humour et que les jeunes lecteurs auraient parfaitement compris qu'il s'agissait là d'une histoire et non d'un modèle à suivre à la lettre. Trop souvent en Malaisie, on a l'impression que l'éditeur se substitue au lecteur et ne lui laisse aucun choix. Doit-on contrôler les lectures d'autrui ?

Un effort important de sensibilisation auprès des éditeurs doit être entrepris tant en France qu'en Malaisie, pour assurer que les textes traduits soient relativement fidèles aux originaux. La traduction est une importation, elle doit être acceptée comme telle et il faut comprendre qu'elle représente, à la longue l'histoire l'a prouvé - un gain pour le pays importateur. Pour sortir du cercle vicieux qui empêche 1'augmentation du nombre de traductions du malais au français, on pourrait promouvoir ces traductions auprès du public. Des fonds devraient être débloqués pour que le public prenne conscience de la grande richesse des littératures mondiales, souvent considérées comme mineures ou secondaires. Une fois le public informé, les traductions d'aires ignorées se feront plus naturellement et seront accueillies, au même titre que les autres. Inversement, il faudrait sans doute agir de concert avec l'Association des traducteurs de Malaisie (Persatuan Penterjemah Malaysia) afin d'avoir de meilleurs rapports avec les éditeurs, autres que le DBP, qui a manifesté très clairement son intérêt pour la traduction, et leur faire accepter que la traduction est un produit importé, qu'il faut respecter et, donc, accepter dans sa totalité.

Pour que progresse la traduction du malais en français et vice-versa - les deux volets de la traduction doivent être équilibrés, pour témoigner du respect des uns et des autres - le projet d'associer l'ESIT à la formation des traducteurs est certainement à encourager. Il faut aussi tenter de créer des vocations, tout en rappelant qu'un traducteur litéraire ne fait fortune nulle part, mais que ce dernier est amplement récompensé quand il apprend que l'œuvre qu'il a choisie, appréciée et traduite a été publiée et jouit d'un certain succès dans le pays d'adoption.

Plus de liberté au traducteur, plus de respect de l'écrit de la part de l'éditeur, plus de formation permanente, plus de campagnes d'information auprès du grand public et la traduction franco-malaise est sauvée!

LAURENT METZGER Université nationale de Singapour, Singapour 